\title{
On the stellar content of the open clusters Melotte 105, Hogg 15, Pismis 21 and Ruprecht 140*
}

\begin{abstract}
A. E. Piatti ${ }^{1, \star \star}$ and J. J. Clariá ${ }^{1}$
Observatorio Astronómico, Laprida 854, 5000 Córdoba, Argentina

Received 28 November 2000 / Accepted 30 January 2001

Abstract. CCD observations in the $B, V$ and $I$ passbands have been used to generate colour-magnitude diagrams reaching down to $V \sim 19$ mag for two slightly characterized (Melotte 105 and Hogg 15) and two almost unstudied (Pismis 21 and Ruprecht 140) open clusters. The sample consists of about 1300 stars observed in fields of about $4^{\prime} \times 4^{\prime}$. Our analysis shows that neither Pismis 21 nor Ruprecht 140 are genuine open clusters since no clear main sequences or other meaningful features can be seen in their colour-magnitude diagrams. Melotte 105 and Hogg 15 are open clusters affected by $E(B-V)=0.42 \pm 0.03$ and $0.95 \pm 0.05$, respectively. Their distances to the Sun have been estimated as $2.2 \pm 0.3$ and $2.6 \pm 0.08 \mathrm{kpc}$, respectively, while the corresponding ages estimated from empirical isochrones fitted to the Main Sequence cluster members are 350 Myr and $300 \mathrm{Myr}$, respectively. The present data are not consistent with the membership of the WN6 star HDE 311884 to Hogg 15.
\end{abstract}

Key words. open clusters: individual: Melotte 105, Hogg 15, Pismis 21, Ruprecht 140 - open clusters: general techniques: photometric

\section{Introduction}

It has long been recognized that open clusters are extremely important in the study of the structure and evolution of the disc of our Galaxy (Friel 1995; Twarog et al. 1997). Particularly, open clusters located towards the Galactic centre play an important role as they offer the possibility of tracing the structure and evolution of the inner disc. Many open clusters in this Galactic area, however, have not been studied in detail yet because they are affected by high interstellar absorption and/or strong field star contamination. A large number of these unstudied objects are certainly included in the $60 \%$ of the approximately 1200 catalogued open clusters which lack a Colour-Magnitude Diagram (CMD) at present (see, http://obswww . unige.ch/webda/).

Thus, we have recently started a systematic survey that consists of obtaining CMDs of numerous, mostly unstudied, open clusters (or candidates), preferably located towards the central parts of the Galaxy (Piatti et al. 1999 and references therein). The main purpose of this long-term programme is to determine whether they are genuine physical systems or if, on the contrary, the apparent enhancement of stars in a sky region is a conse-

Send offprint requests to: A. E. Piatti,

e-mail: andres@mail .oac. uncor.edu

* Tables 2 to 5 are only available in electronic form at the CDS via anonymous ftp to

cdsarc.u-strasbg.fr $(130.793 .128 .5)$ or via

http://cdsweb.u-strasbg.fr/cgi-bin/qcat?J/A+A/370/931

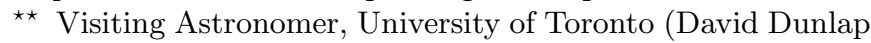
Observatory) $0.6 \mathrm{~m}$ telescope, Las Campanas, Chile. quence of random fluctuations of the stellar density in that zone. The CMDs also allow us to determine reddenings, distances and ages of those objects confirmed as open clusters, and therefore, to improve our knowledge of the structure and evolution of the inner Galactic disc, with clusters distributed along a larger baseline in galactocentric distances (Piatti et al. 1995; Piatti 1997).

Open cluster candidates were first selected from the Alter et al. (1970) and Lyngå (1987) open cluster catalogues. Some candidates were then eliminated since they already had associated photometric and/or spectroscopic studies. To facilitate the study through CCD observations, we assigned a higher priority to objects with angular sizes smaller than $4^{\prime}$, thus including in our sample most of the stars concentrated in the regions of interest. In order to avoid saturation or low signal-to-noise ratios, we also took into account the apparent brightness of the clusters. The brightest stars in each object have $V \sim 12-14 \mathrm{mag}$, according to the open cluster catalogues mentioned above. Notice that some objects selected from Alter et al. (1970) could not be real clusters, since many of them were included in the catalogues based on their visual appearance in photographic plates. A visual photographic inspection of these objects reveals, in general, the presence of star condensations which are hard to distinguish from the background. These stellar density differences might be caused by an accidental star distribution along the line of sight, by the presence of irregularly distributed interstellar material, by the presence of a genuine cluster, or by some combination of these three factors. Finally, using the ESO/SRC Sky Atlas $\mathrm{J}$ and $\mathrm{R}$ plates, we rejected some candidates for any one of the following reasons: they are located near a very 
bright star, or within HII regions; they are small concentrations very difficult to identify, or they have a small number of sparse stars.

In this paper we present the results obtained for the following selected objects: Melotte 105 (OCI-856, $\alpha_{1950}=$ $\left.11^{\mathrm{h}} 17^{\mathrm{m}} 3, \quad \delta_{1950}=-63^{\circ} 14^{\prime} ; l=292.89, b=-2.45\right)$, Hogg 15 (OCI-891, $\alpha_{1950}=12^{\mathrm{h}} 40^{\mathrm{m}} 6, \delta_{1950}=-62^{\circ} 50^{\prime}$; $l=302.04, b=-0.24$ ), Pismis 21 (OCI-931, $\alpha_{1950}=$ $\left.15^{\mathrm{h}} 12 \mathrm{~m} 8, \delta_{1950}=-59^{\circ} 28^{\prime} ; l=320.36, b=-1.79\right)$ and Ruprecht 140 (OCI-1, $\alpha_{1950}=18^{\mathrm{h}} 18^{\mathrm{m}} \cdot 5, \delta_{1950}=-33^{\circ} 12^{\prime}$; $l=0.01, b=-8.86)$. The published data on these clusters are rather heterogeneous. Melotte 105 is the only cluster in the sample with several previous studies. $U B V$ photoelectric and photographic observations were carried out by Sher (1965) for a total of 85 stars in the cluster field. More recently, Kjeldsen \& Frandsen (1991) performed CCD photometry in the same photometric system for 127 stars. The cluster was also target of uvby CCD photometric (Balona \& Laney 1995) and integrated spectroscopic (Santos \& Bica 1993; Ahumada et al. 2000) studies. Reddening, distance and age values derived from these studies vary with respect to their mean values around 50, 20, and 80\%, respectively. Moffat (1974) also derived fundamental parameters of Hogg 15 using $U B V$ photoelectric photometry of 23 stars located in the cluster field, whereas Orsatti et al. (1998) concluded that some "intracluster" dust exists in association with Hogg 15, based on $U B V R I$ polarimetric observations of these 23 stars. Moffat \& FitzGerald (1977) reported $U B V$ photometry of 11 stars in the field of Pismis 21, one of them being the F8I supergiant HD 135159. Their photometry indicates that HD 135159 is surrounded by at least six early B stars with variable extinction: $\langle E(B-V)\rangle=$ $1.66 \pm 0.13$ (s.d.), which are located at a distance of $\sim 1.7$ kpc. Ruprecht 140, as far as we know, has not been studied.

This paper is structured as follows: in Sect. 2 the observations are presented and the reduction procedure is briefly described. The analysis of the cluster CMDs is described in Sect. 3, while the cluster parameters are derived in Sect. 4. Finally, the conclusions of this work are given in Sect. 5 .

\section{Observations and reductions}

CCD BVI images for the fields of Melotte 105, Hogg 15 and Pismis 21 were taken during an observing run in June/July 1995 with the University of Toronto Southern Observatory $0.6 \mathrm{~m}$ telescope (Las Campanas Observatory, Chile) and with a PM $512 \times 512$ METACHROME chip coated to give improved blue response. The scale on the chip was $0.45^{\prime \prime}$ per pixel, yielding an area covered by a frame of $4^{\prime} \times 4^{\prime}$. Ruprecht 140 was observed during a previous observing run in September 1994 with the same telescope and instrument. The air-masses of the observed fields were always less than 1.35, just lying within the air-mass range of the standard stars, and the seeing was typically $1.6^{\prime \prime}$. The observations were supplemented with nightly exposures of 10 bias, 10 dome- and 10 twilight sky-flats to calibrate the CCD instrumental signature. We also observed nightly an average of 12 standard stars of the Selected Areas \# 104, 105, 107, 108, 110, 111, 112 and 113 (Landolt 1992) covering a wide range in colour $(-0.1 \leq V-I \leq 2.0)$ to transform the instrumental magnitudes into the standard system. Table 1 lists the exposure sequences and seeing for each object and filter, while Fig. 1 shows the schematic finding chart built with all the stars measured in the $V$ band.

The IRAF $^{1} /$ DAOPHOT package was used to reduce the observations in the standard way at the Astronomical Observatory of the National University of Córdoba, Argentina. The $b, v$ and $i$ instrumental magnitudes (corrected to $1 \mathrm{~s}$ integration using a zero point of $25.0 \mathrm{mag}$ ) for a total of 1311 stars were transformed into the standard system using the following equations:

$$
\begin{aligned}
& b_{j, n}=b_{1}+V+(B-V)+b_{2} \times(B-V)+b_{3} \times X_{j, n} \\
& v_{k, n}=v_{1}+V+v_{2} \times(B-V)+v_{3} \times X_{k, n} \\
& i_{l, n}=i_{1}+V-(V-I)+i_{2} \times(V-I)+i_{3} \times X_{l, n}
\end{aligned}
$$

where $V,(B-V)$ and $(V-I)$ are the standard magnitude and colours and $X$ the corresponding air-mass for the $j, k, l$ th measured standard star. We solved the equations for all coefficients simultaneously for each night $n$ with the PHOTCAL package in IRAF. The nightly rms errors from the transformation into the standard system ranged from 0.004 to $0.014 \mathrm{mag}$ in $B, 0.005$ to $0.028 \mathrm{mag}$ in $V$ and 0.009 to $0.029 \mathrm{mag}$ in $I$, with means of $0.007,0.012$ and $0.015 \mathrm{mag}$, respectively, which indicate that the nights were all of good photometric quality. Figure 2 shows the trend of the photometric magnitude and colour errors with $V$ provided by DAOPHOT for the richest cluster of the sample (see Sect. 3). For each selected field we generated a master table containing a running star number, the $X$ and $Y$ coordinates, the $V$ magnitude, the $B-V$ and $V-I$ colours, the observational errors provided by the INVERTFIT task $\sigma(V)$, $\sigma(B-V)$, and $\sigma(V-I)$ and the number of observations $n$. These tables were built by combining all the independent measurements using the stand-alone DAOMATCH and DAOMASTER programmes kindly provided by Peter Stetson. Tables 2 to 5 provide this information and are available in electronic form at the CDS via anonymous ftp to cdsarc.u-strasbg.fr.

A comparison of our CCD photometry with previous photoelectric measurements carried out in Melotte 105 and Hogg 15 shows good agreement. For three stars observed in common with Sher (1965) in Melotte 105, the mean differences $(\Delta=$ Sher-CCD) are: $\Delta V=-0.03 \pm 0.03$ and $\Delta(B-V)=-0.01 \pm 0.05$, while for 16 stars measured in common with Moffat (1974) in Hogg 15, these

\footnotetext{
${ }^{1}$ IRAF is distributed by the National Optical Astronomy Observatories, which is operated by the Association of Universities for Research in Astronomy, Inc., under contract with the National Science Foundation.
} 


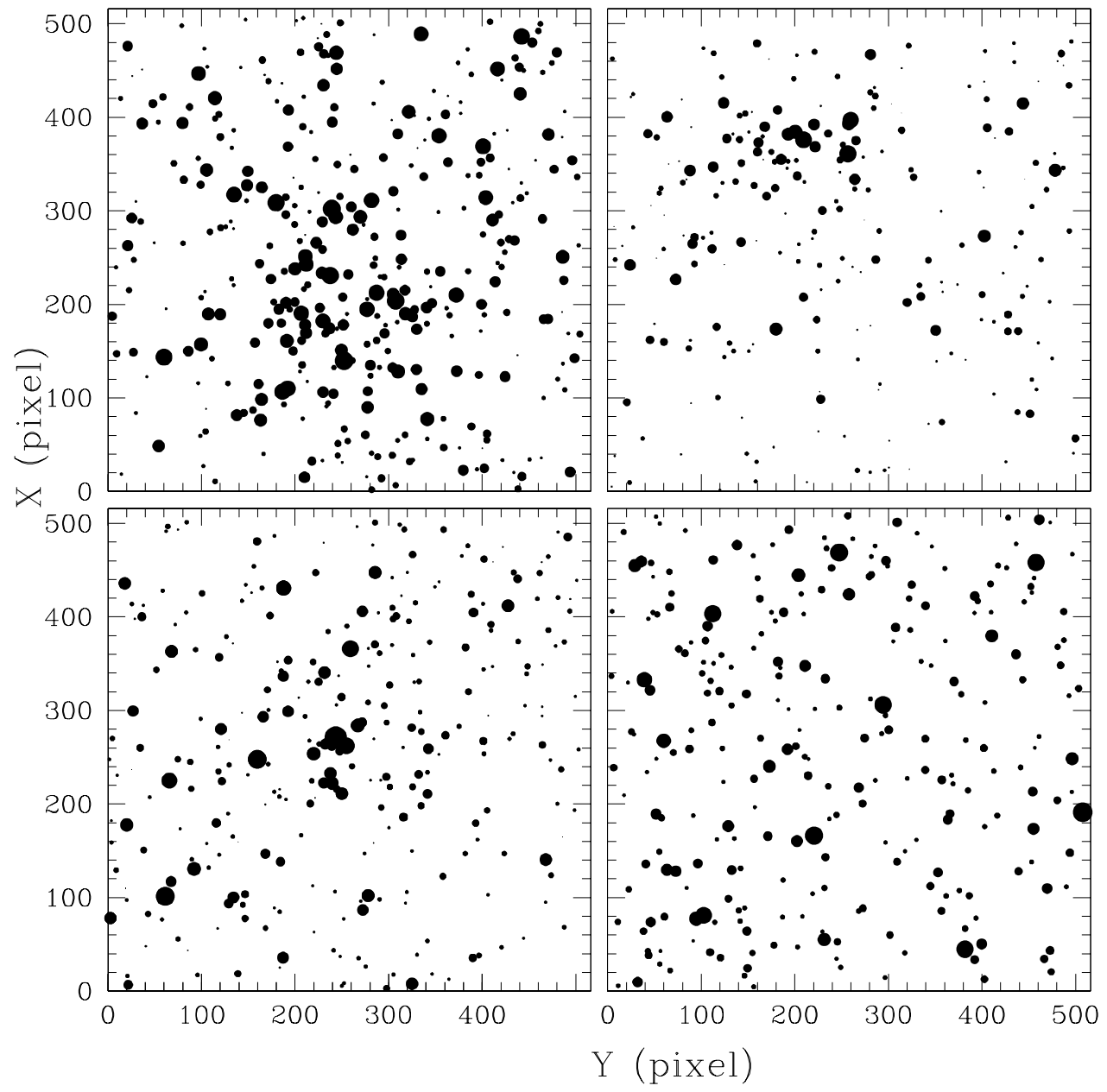

Fig. 1. Schematic finding charts for the fields of Melotte 105 (top-left), Hogg 15 (top-right), Pismis 21 (bottom-left), and Ruprecht 140 (bottom-right). North is up and East is to the left. The sizes of the plotting symbols are proportional to the brightness of the stars

Table 1. Journal of observations

\begin{tabular}{lcccc}
\hline Object & $\begin{array}{c}\text { Date } \\
\text { (UT) }\end{array}$ & Filter & Exposures & $\begin{array}{c}\text { seeing } \\
\left({ }^{\prime \prime}\right)\end{array}$ \\
\hline Melotte 105 & 1995 June 29 & $B$ & $1 \times 60 \mathrm{~s}, 2 \times 300 \mathrm{~s}$ & 2.0 \\
& & $V$ & $1 \times 60 \mathrm{~s}, 1 \times 120 \mathrm{~s}, 1 \times 150 \mathrm{~s}$ & 2.0 \\
Hogg 15 & \multirow{2}{*}{ 1995 June 27 } & $B$ & $2 \times 30 \mathrm{~s}, 1 \times 40 \mathrm{~s}$ & 2.0 \\
& & $V$ & $1 \times 60 \mathrm{~s}, 1 \times 600 \mathrm{~s}, 1 \times 900 \mathrm{~s}$ & 1.7 \\
& & $I$ & $1 \times 60 \mathrm{~s}, 2 \times 300 \mathrm{~s}$ & 1.6 \\
Pismis 21 & 1995 July 2 & $B$ & $1 \times 60 \mathrm{~s}, 2 \times 300 \mathrm{~s}$ & 1.3 \\
& & $V$ & $1 \times 20 \mathrm{~s}, 1 \times 120 \mathrm{~s}, 1 \times 180 \mathrm{~s}$ & 1.5 \\
Ruprecht 140 & 1994 September 4 & $B$ & $1 \times 60 \mathrm{~s}, 2 \times 300 \mathrm{~s}$ & 1.9 \\
& & $V$ & $1 \times 60 \mathrm{~s}, 2 \times 100 \mathrm{~s}$ & 1.3 \\
& & $I$ & $1 \times 10 \mathrm{~s}, 2 \times 15 \mathrm{~s}$ & 1.3 \\
\hline
\end{tabular}

differences are: $\Delta V=+0.06 \pm 0.12$ and $\Delta(B-V)=$ $+0.00 \pm 0.07$. These values show that there are no significant offsets in our CCD zero-point magnitude and colour scales. We have also compared our CCD photometry with that of Kjeldsen \& Frandsen (1991) for 125 stars in common, the mean raw differences being: $\Delta V=0.02 \pm 0.05$ and $\Delta(B-V)=0.01 \pm 0.07$. These authors noted an observational offset of 0.07 between their $B-V$ values and those of Sher (1965). However, there is not only a zeropoint offset in their photometry with respect to that of Sher (and therefore with respect to our CCD photometry), 


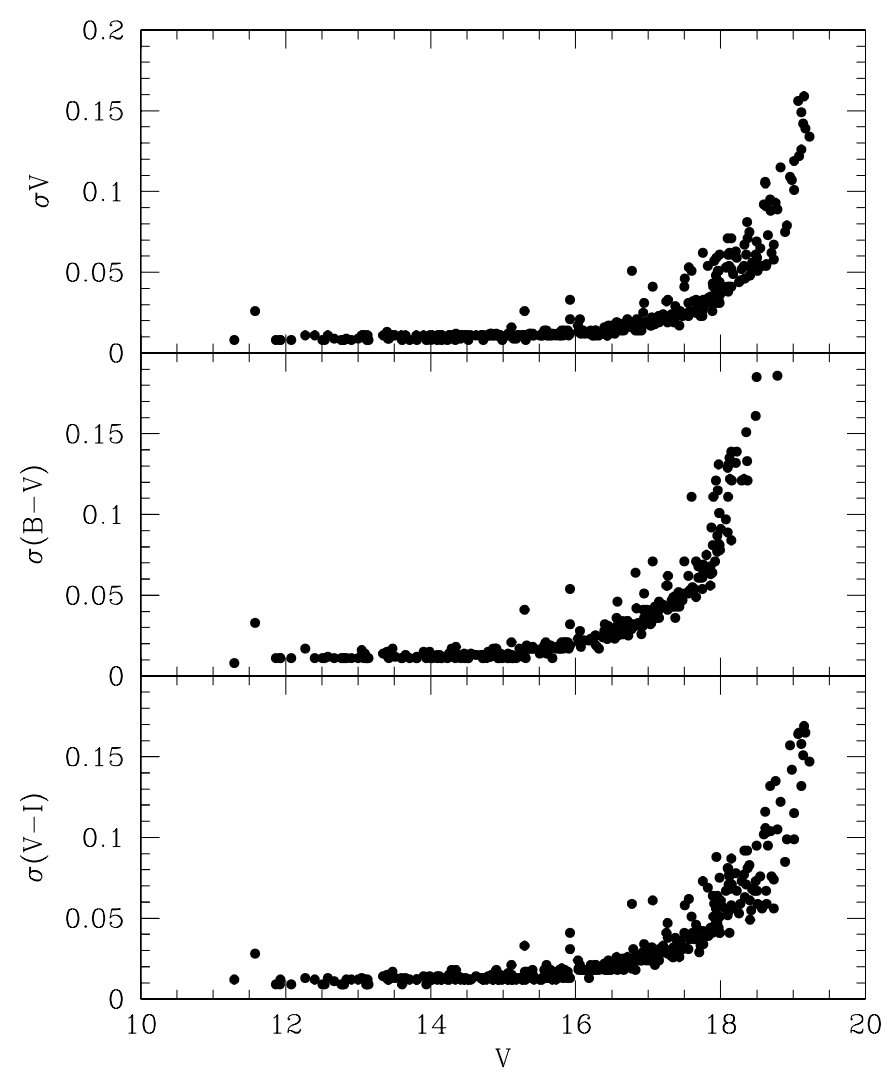

Fig. 2. Magnitude and colour photometric errors provided by DAOPHOT as a function of $V$ for the richest cluster of the sample (Melotte 105). They are typical in our sample

but also a peculiar behaviour of the mean differences and dispersions with $V$ and $B-V$, as shown in Fig. 3 .

\section{Colour-magnitude diagram analysis}

Since the selected objects were first identified in old catalogues, we decided to use our CCD finding charts (Fig. 1) to examine their star distributions. The angular sizes for Melotte 105, Hogg 15, Pismis 21 and Ruprecht 140 given by Alter et al. (1970) are 4..0, 2..0, 2..0 and 3.5, respectively, so that our CCD frames should cover almost their entire fields. Indeed, Melotte 105 seems to extend over the whole CCD area, while Hogg 15 appears as a somewhat elongated group of bright and faint stars in the upperleft part of the chart. Pismis 21 seems to be a handful of concentrated stars dominated by some bright stars at the centre of the image. This would not appear to be the case for Ruprecht 140, for which we did not recognize any clear star concentration. According to its coordinates (Lyngå $1987)$, its centre is placed at $\left(X_{\mathrm{c}}, Y_{\mathrm{c}}\right) \approx(350,100)$, where there are some faint stars with a halo of bright stars at $\sim 2^{\prime}$ around them. We presume that these stars might have appeared like an open cluster in old field photographic plates. Although Ruprecht (1966) classified the star richness of all these objects as poor, Fig. 1 shows that the star density considerably varies from one field to another. In fact, in the fields of Melotte 105 and Hogg 15, 356 and 343 stars have been measured, respectively, while in the fields of

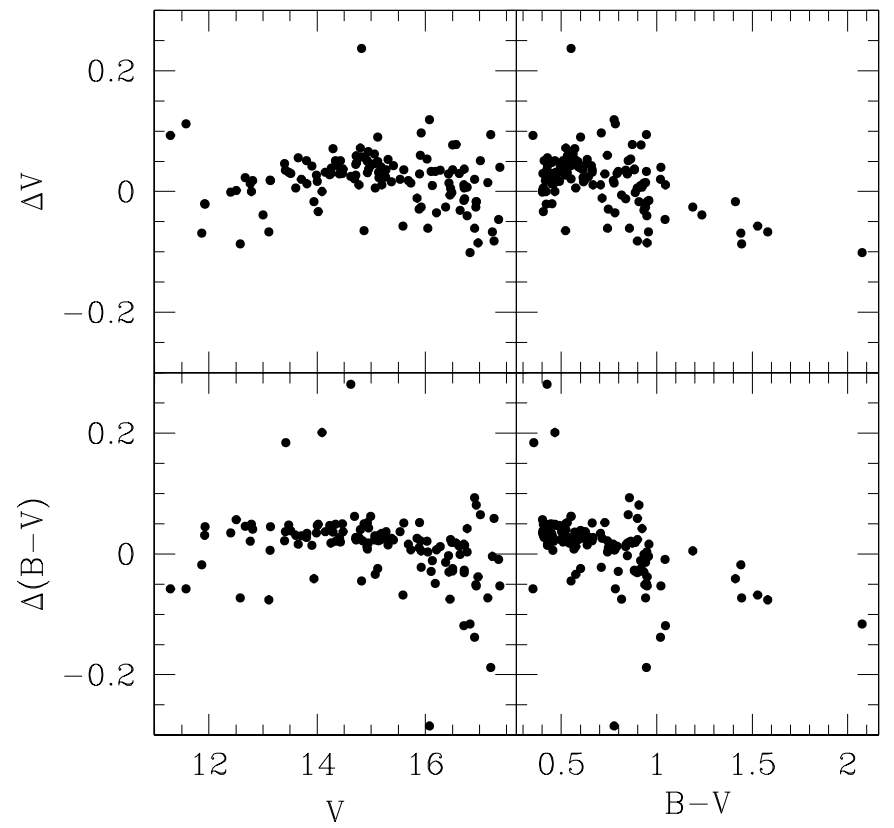

Fig. 3. Magnitude and colour differences between Kjeldsen \& Frandsen (1991) and our values for Melotte 105 as a function of $V$ and $B-V$

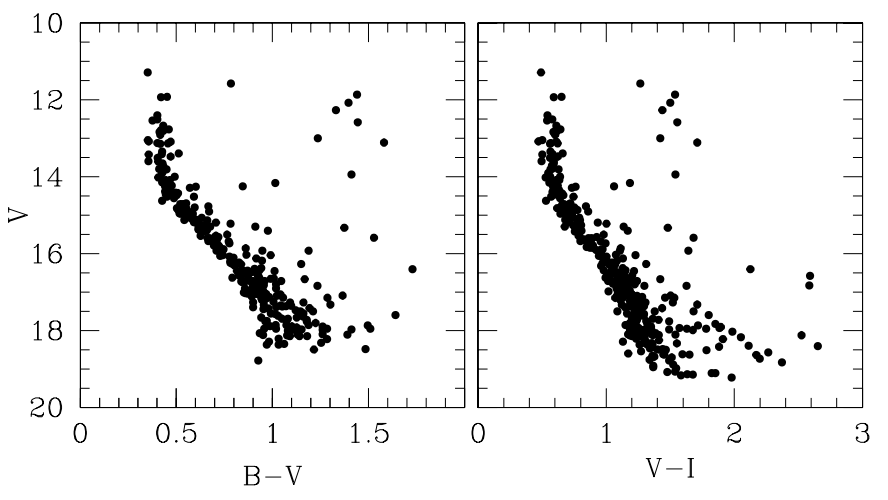

Fig. 4. $(V, B-V)$ and $(V, V-I)$ colour magnitude diagrams for stars observed in the field of Melotte 105

Pismis 21 and Ruprecht 140 nearly 50\% fewer stars have been measured. Thus, Melotte 105 is the richest cluster of the present sample.

Figure 4 shows the resulting CMDs for Melotte 105. The Main Sequence (MS) of a moderately young open cluster is clearly visible, which extends down to $V \sim$ $18.5 \mathrm{mag}$ and covers a range of $\Delta V \sim 7 \mathrm{mag}$. Our $(V$, $B-V)$ CMD, even when obtained using a telescope with an aperture 2.5 times smaller than that used by Kjeldsen \& Frandsen (1991), results in $\sim 1.5$ mag deeper than their CMD. Notice also that the present photometry almost triples the number of stars measured by Kjeldsen \& Frandsen, thus superseding any previous photometric study on this cluster. The cluster seems to be affected neither by a high reddening nor by field star contamination, as judged from the similar limiting magnitude reached in both CMDs and the scarce number of field stars lying outside the MS. The star with $V=11.291, B-V=0.351$ 


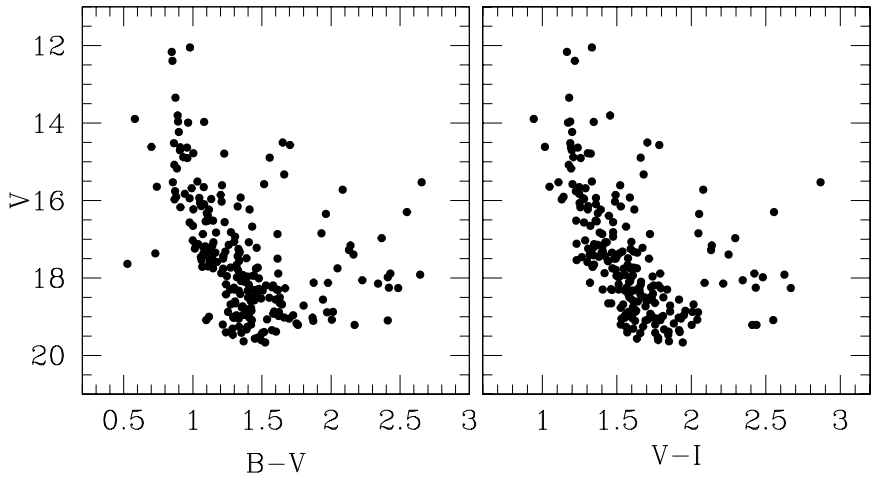

Fig. 5. $(V, B-V)$ and $(V, V-I)$ colour magnitude diagrams for stars observed in the field of Hogg 15

and $V-I=0.491$ in the upper-left of the MS is suspected to be a blue straggler. Ahumada \& Lapasset (1995) included this star in the third class of their catalogue of blue stragglers in open clusters, since it is located in the blue straggler area but has no membership indicator.

The CMDs for stars measured in the field of Hogg 15 are shown in Fig. 5. Like Melotte 105, the cluster MS extends through a large magnitude range. The upper MS looks tighter than its lower part, and it is mostly composed of the brightest stars in the field, which are clearly concentrated within a circle $\leq 22^{\prime \prime} .5$ (50 pixels) around the adopted cluster centre $\left(X_{\mathrm{c}}, Y_{\mathrm{c}}\right)=(380,230)$. The lower MS becomes broader than the upper MS, particularly for $V \leq 16$ mag. Furthermore, the lower MS remains somewhat wider than the upper MS, even if we removed some probable field stars placed towards the reddest side of the cluster's lower MS. This broadness could be caused by differential reddening, since the cluster is near the centre of the Coalsack dark cloud. NGC 4609 is a well-known open cluster located $\sim 5^{\prime}$ northeast following Hogg 15 , with a spread of the stars around the MS larger than the typical dispersion in other open clusters, probably due to the fact that the distribution of the absorption material inside the Coalsack is far from being uniform (Feinstein \& Marraco 1971; Straiz̃ys et al. 1994). According to Orsatti et al. (1998), the magnetic field in the direction to Hogg 15 follows the general trend of the polarization directions in the region.

The field of Pismis 21 is rather different. Some different structures can be identified in Fig. 6, none of which are similar to an open cluster MS. Ng et al. (1996) studied the distribution of stars from the disc population in CMDs of star fields located at low Galactic latitudes towards the Galactic centre. According to their findings, the slightly tilted star sequence following the direction $V=8 \times(V-I)+7.2$ in Fig. 6 is composed of MS disc stars. Note that each feature in the $(V, V-I)$ CMD has its counterpart in the $(V, B-V)$ CMD. This sequence becomes approximately one magnitude fainter than in the $(V, B-V) \mathrm{CMD}$ as a result of the contribution of redder stars. Moreover, the number of stars fainter than $V \sim 18.0 \mathrm{mag}$ in the $(V, V-I) \mathrm{CMD}$ is practically the

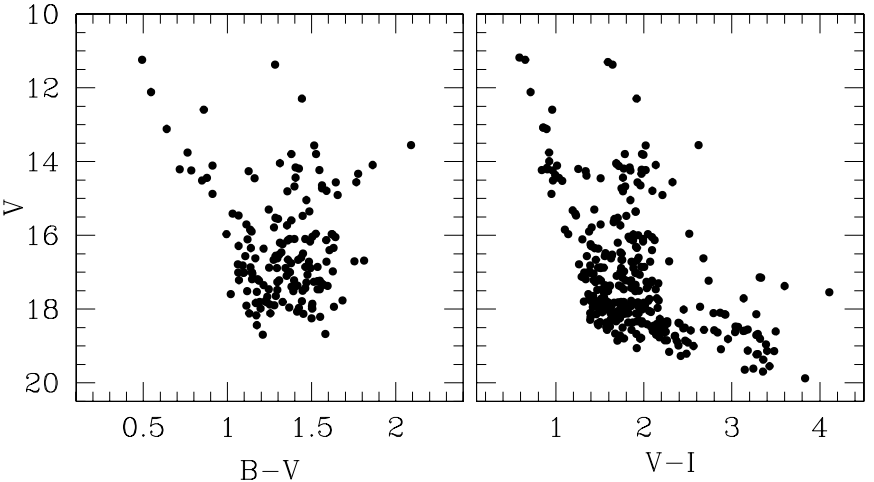

Fig. 6. $(V, B-V)$ and $(V, V-I)$ colour magnitude diagrams for stars observed in the field of Pismis 21

same as that for the total magnitude range in the $V$ vs. $B-V$ CMD. On the other hand, the broad and somewhat dispersed group of stars centered at $(V, V-I) \sim(14.5$, 2.0 ) would seem to correspond to Horizontal Branch (HB) young disc stars, whereas those stars distributed along the sequence placed below this group would be $\mathrm{HB}$ and Red Giant Branch (RGB) old disc stars. Thus the field of Pismis 21 does not correspond to an open cluster field, but to field stars belonging to different disc components.

Carraro \& Patat (1995) concluded that Ruprecht 46, also catalogued as an open cluster, does not form a physical system. Their CMDs reflect the characteristics of the Galactic field across the line of sight. The star sequence in the CMDs of Ruprecht 46 nearly follows the direction of the tilted sequence in the Pismis 21 CMDs. Notice that the position and dispersion of the field star sequences also depend on interstellar absorption through the various Galactic directions. However, not every loose open cluster candidate always results in a random fluctuation of the star density in a region of the sky. This is the case, for example, for NGC 7762: a scarcely populated open cluster whose appearance in the $V$ image is not substantially different from that of Pismis 21 (Patat \& Carraro 1995). This result demonstrates that the apparent visual identification of cluster candidates is not at all a sufficient criterion to assess their physical reality. Based on the assumption that Pismis 21 is a real cluster, Moffat \& FitzGerald (1977) estimated a distance from the Sun of $1.7 \mathrm{kpc}$ and Ahumada et al. (2000) derived a reddening value, age and metallicity for the stars located at the centre of Fig. 1 from integrated spectroscopy. However, we want to stress that this technique is very effective when it is used as a complementary tool, preferably of confirmed compact clusters.

Ruprecht 140, from the inspection of its CMDs in Fig. 7, is not an open cluster and, perhaps, some haziness produced by faint stars in low spatial resolution photographic plates with some bright stars around it could have caused its identification as an open cluster candidate.

\section{Cluster parameters}

In order to derive reddening, distance modulus and age of Melotte 105 and Hogg 15, we first matched the $(V, B-V)$ 


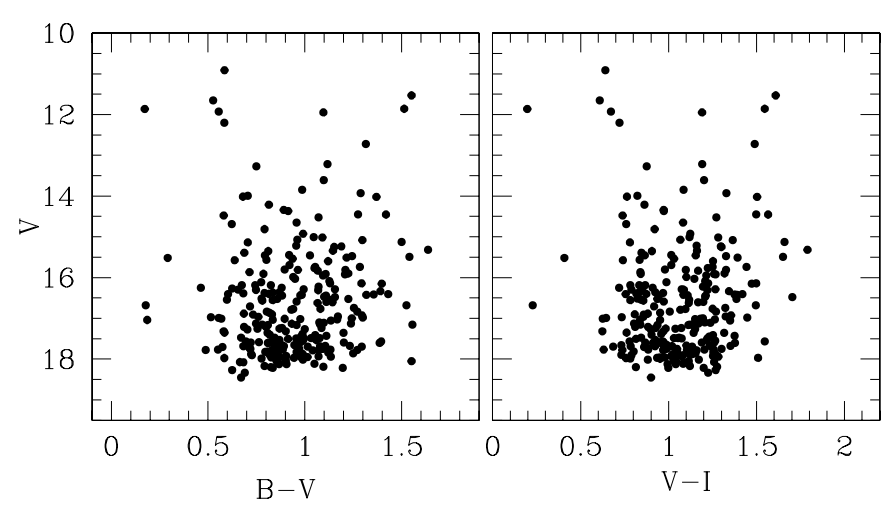

Fig. 7. $(V, B-V)$ and $(V, V-I)$ colour magnitude diagrams for stars observed in the field of Ruprecht 140

and $(V, V-I)$ CMDs to the Zero-Age Main Sequences (ZAMSs) of Schmidt-Kaler (1982) and Piatti et al. (1998), independently. We thus obtained for each cluster two apparent distance moduli $V-M_{v}$ and two different colour excess $E(B-V)$ and $E(V-I)$ values. The errors in these quantities were estimated from the observed dispersion of MS stars. The average ratio $E(V-I) / E(B-V)$ resulted in $1.28 \pm 0.05$, which is in very good agreement with the value 1.25 generally adopted for the normal extinction law (Walker 1985; Straiz̃ys 1990). Adopting 3.0 for the ratio $R=A_{v} / E(B-V)$ of total to selective absorption, the true distance moduli were then derived for each cluster. Table 6 lists the resulting cluster parameters. The distance error was computed with the expresion: $\sigma(d)=0.46 \times\left[\sigma\left(V-M_{v}\right)+3 \times \sigma(E(B-V))\right] \times d$, where $\sigma\left(V-M_{v}\right)$ and $\sigma(E(B-V))$ represent the estimated errors in $V-M_{v}$ and $E(B-V)$, respectively. Figures 8 and 9 show the $M_{v}$ vs. $(B-V)_{0}$ and $M_{v}(V-I)_{0}$ diagrams for Melotte 105 and Hogg 15, respectively. The ZAMS of Schmidt-Kaler (1982) and Piatti et al. (1998) have been adjusted to the adopted distance moduli in each cluster. Finally, cluster ages were derived by matching the $(V$, $V-I)$ CMDs onto the empirical isochrones traced by Piatti et al. (1998) in the $\left(M_{v},(V-I)_{0}\right)$ plane. For that purpose, we used the mean reddening values and apparent distance moduli obtained previously. As shown in Fig. 10, both clusters seem to be moderately young.

As far as Melotte 105 is concerned, both the colour excess $E(B-V)$ and the distance obtained in this study are in good agreement with the values derived by Sher (1965), i.e. 0.38 and $2.1 \mathrm{kpc}$, respectively. On the other hand, although Kjeldsen \& Frandsen (1991) derived a distance similar to ours, the colour excess $E(B-V)$ they obtained differs more than three times the error we estimated $(\Delta E(B-V)=0.09)$. Indeed, the offset of 0.07 mags in the $(B-V)$ colours found by Kjeldsen \& Frandsen (1991) between their photometry and that of Sher (1965) explains, at least partially, the differences found in $E(B-V)$. According to the present results, however, Melotte 105's age (350 Myr) is somewhat greater than those derived by Kjeldsen \& Frandsen (1991) and Sher (1965), i.e. $150 \mathrm{Myr}$ and 100 Myr, respectively. Balona \& Laney (1995) also derived colour excess and distance similar to our values using CCD Strömgren photometry. They obtained $E(b-y)=0.34$, equivalent to $E(B-V)=0.46$ if the relation $E(B-V)=1.35 E(b-y)$ derived by Crawford (1978) is used, and $V_{0}-M_{v}=11.31 \pm 0.08$, equivalent to $1.8 \mathrm{kpc}$. Using their $E(b-y)$ value, they estimated an age of $250 \pm 100 \mathrm{Myr}$ for the cluster, closer to the one derived here. Balona \& Laney (1995) explicitly suggested that a lower reddening produces a better agreement with an isochrone of 400 Myr. Indeed, as their Fig. 11 shows, the theoretical ZAMS does not fit the lower envelope of the cluster MS but rather the upper one. If we lower the $E(b-y)$ reddening by $\approx 0.03 \mathrm{mag}$, then the cluster MS moves redwards, the fitting improves and the equivalent $E(B-V)$ colour excess, distance modulus and age turn out to be in very good agreement with our present estimates. On the other hand, Santos \& Bica (1993) and Ahumada et al. (2000) used integrated spectra to derive reddening and age for Melotte 105. Both studies agree that the reddening is approximately $0.3 \mathrm{mag}$ and that the cluster is 200 Myr. Santos \& Bica (1993) and Ahumada et al. (2000) argue that the lower colour excess obtained from integrated spectroscopy probably reflects the existence of internal dust associated to the cluster. However, neither the estimated age for this object nor the apparent width of the MS seen in the observed CMDs seem to be compatible with the probable existence of differential reddening within the cluster. We believe that the difference of scarcely more than 0.1 mag between the spectroscopic and the photometric reddenings is most probably due to the fact that the bright blue straggler $(V=11.29)$ contributes to the integrated light with a greater flux than the remaining cluster stars. We would like to note that the integrated spectral library used by Santos \& Bica (1993) and Ahumada et al. (2000) have template spectra of 100 and $500 \mathrm{Myr}$, but no template in the 100-500 Myr range is yet available. Finally, according to Ahumada \& Lapasset (1995) we should expect one blue straggler on average, which is in very good agreeement with the number of stars found in the blue straggler area of the CMDs of Melotte 105.

A comparison of the derived fundamental parameters of Hogg 15 with those given by Moffat (1974) reveals some discrepancies. Indeed, based on photoelectric photometry of only 15 MS stars, Moffat (1974) reached the conclusion that Hogg 15 is a very young cluster $(8 \mathrm{Myr})$, reddened by $E(B-V)=1.16$ and located in the inner arm-II at a distance of $4.2 \mathrm{kpc}$ from the Sun. By contrast, the present study, based on the CCD photometry of approximately 200 probable MS stars, shows that Hogg 15 is a much older cluster (300 Myr), located at a significantly shorter distance $(d=2.6 \mathrm{kpc})$. Undoubtedly, the significant differences in age as well as distance, stem from the fact that Moffat included star WN6 HDE 311884 as a likely member of Hogg 15 with $M_{v}=-5.9$. This star, however, lies outside the cluster radius estimated by Moffat (see his Fig. 1) at a distance where the cluster star density drops off more than 8 times the central cluster star density, just close to 


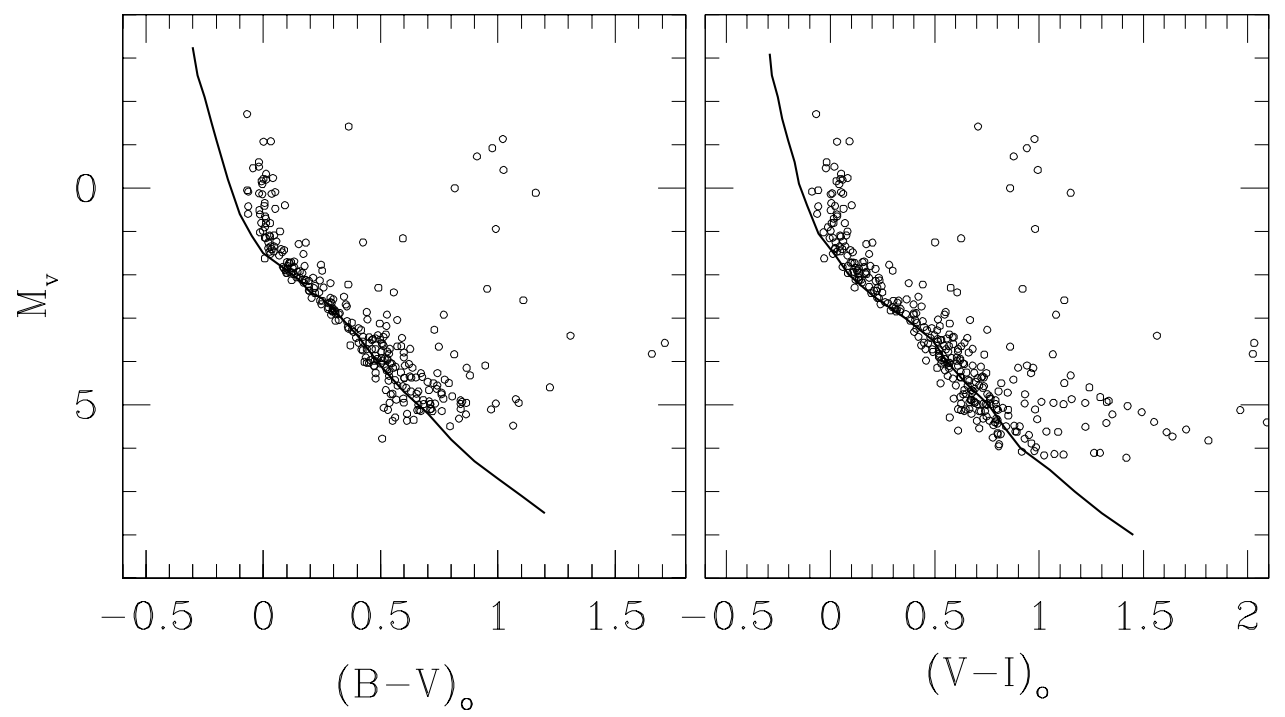

Fig. 8. $\left(M_{v},(B-V)_{0}\right)$ and $\left(M_{v},(V-I)_{0}\right)$ colour magnitude diagrams for stars in the field of Melotte 105. The solid lines represent the ZAMSs of Schmidt-Kaler (1982) and Piatti et al. (1998), respectively

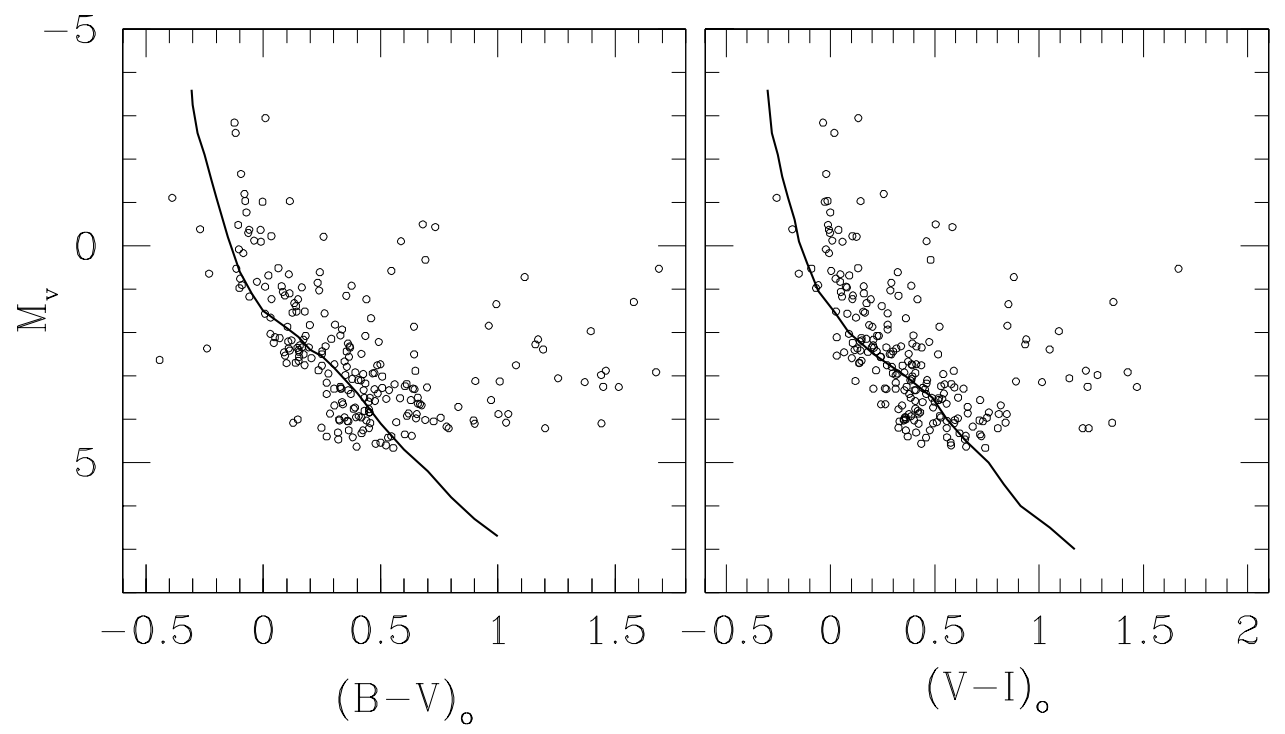

Fig. 9. $\left(M_{v},(B-V)_{0}\right)$ and $\left(M_{v},(V-I)_{0}\right)$ colour magnitude diagrams for stars in the field of Hogg 15 . The solid lines represent the ZAMSs of Schmidt-Kaler (1982) and Piatti et al. (1998), respectively

the background star level. The differences in the $E(B-V)$ values derived can be attributed to the different number of MS stars considered in either study and, probably, also to the fact that the interstellar material is not uniformly distributed in the direction towards the cluster. Based on integrated spectroscopy, Ahumada et al. (2000) derived a reddening of $1.05 \pm 0.05$ in better agreement with our estimate and an age of $5 \pm 2 \mathrm{Myr}$. The significant age difference most probably arises from the contribution of the bright star HDE 311884 to the integrated light.

\section{Conclusions}

We have presented CCD BVI Johnson-Cousins photometry of stars in the fields of the open clusters Melotte 105, Hogg 15, Pismis 21 and Ruprecht 140, all of them located towards the central parts of the Galaxy. The photometric data on Melotte 105, Hogg 15 and Pismis 21 supersede previous measurements in these clusters, thus allowing us to extend the corresponding MSs down to $V \sim 19$.

Melotte 105 and Hogg 15 were found to be genuine open clusters, while Pismis 21 and Ruprecht 140 appeared not to be open clusters but simple density fluctuations above the background level. The average ratio $E(V-$ $I) / E(B-V)$ derived for the two genuine clusters was found to be $1.28 \pm 0.05$, in very good agreement with the value 1.25 generally adopted for the normal extinction law. The present photometric data indicate that Melotte 105 and Hogg 15 are moderately young (300-350 Myr) open clusters, located approximately in the direction towards the Galactic centre at $2.2 \mathrm{kpc}$ and $2.6 \mathrm{kpc}$ from the Sun, respectively. The difference between the photometric 


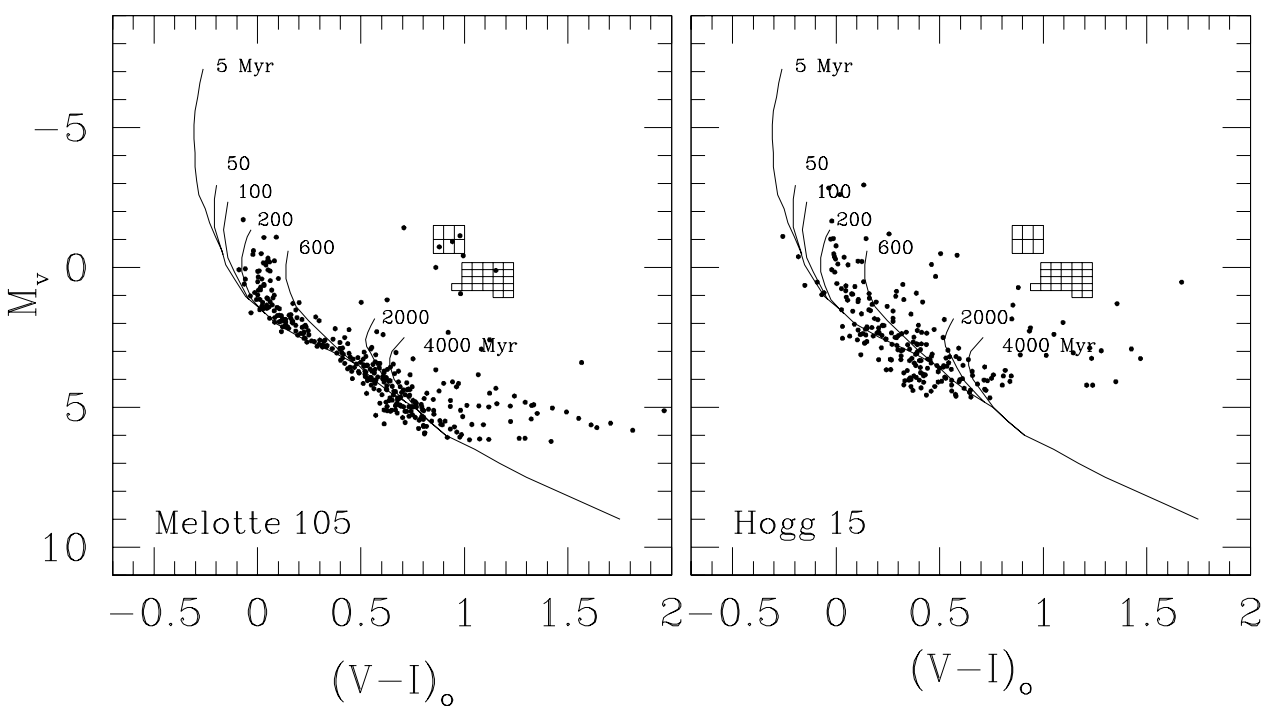

Fig. 10. $\left(M_{v},(V-I)_{0}\right)$ colour magnitude diagrams (filled circles) with the isochrones of Piatti et al. (1998) superimposed. The big and small squares represent the position of the red giant clumps of NGC 6259 (age $\approx 0.2$ Gyr) and NGC 3680 and IC 4651 (age $\approx 2.0 \mathrm{Gyr}$ ), respectively

Table 6. Adopted cluster fundamental parameters

\begin{tabular}{lccccc}
\hline Cluster & $E(B-V)$ & $E(V-I)$ & $V_{0}-M_{v}$ & $\begin{array}{c}d \\
(\mathrm{kpc})\end{array}$ & $\begin{array}{c}\text { Age } \\
(\mathrm{Myr})\end{array}$ \\
\hline Melotte 105 & $0.42 \pm 0.03$ & $0.57 \pm 0.03$ & $11.75 \pm 0.25$ & $2.2 \pm 0.3$ & $350 \pm 50$ \\
Hogg 15 & $0.95 \pm 0.05$ & $1.20 \pm 0.05$ & $12.10 \pm 0.50$ & $2.6 \pm 0.8$ & $300 \pm 80$ \\
\hline
\end{tabular}

reddening in Melotte 105 and the one previously determined from integrated spectroscopy by Santos \& Bica (1993) and Ahumada et al. (2000), is most probably due to the fact that the bright blue straggler $(V=11.29)$ contributes to the integrated light with a larger flux than those of the remaining cluster members. On the other hand, the present CCD data are not consistent with the membership of the WN6 star HDE 311884 to Hogg 15, which explains why this cluster is now found to be much older and closer to the Sun than previously thought.

Acknowledgements. We wish to thank the Director, staff and technicians of Las Campanas Observatory for the allocation of observing time and for their kind assistance and hospitality. Thanks are also due to an anonymous referee for making useful suggestions which improved this paper. We gratefully acknowledge financial support from the Argentinian institutions CONICET, Agencia Nacional de Promoción Científica y Tecnológica (ANPCyT) and SECYT (National University of Córdoba).

\section{References}

Ahumada, J., \& Lappaset, E. 1995, A\&AS, 109, 375

Ahumada, A. V., Clariá, J. J., Bica, E., \& Piatti, A. E. 2000, A\&AS, 141, 79

Alter, G., Ruprecht, J., \& Vanýsek, J. 1970, Catalogue of Star Clusters and Associations, ed. G. Alter, B. Balázs, \& J. Ruprecht (Akademiai Kiado, Budapest)

Balona, L., \& Laney, C. 1995, MNRAS, 277, 250
Carraro, G., \& Patat, F. 1995, MNRAS, 276, 563

Crawford, D. L. 1978, AJ, 83, 48

Feinstein, A., \& Marraco, H. G. 1971, PASP, 83, 218

Friel, E. D. 1995, ARA\&A, 33, 381

Kjeldsen, H., \& Frandsen, S. 1991, A\&AS, 87, 119

Landolt, A. U. 1992, AJ, 104, 340

Lyngå, G. 1987, Catalogue of Open Clusters Data, distributed by Centre de Données Stellaires, Strasbourg

Moffat, A. F. J. 1974, A\&A, 34, 29

Moffat, A. F. J., \& FitzGerald, M. P. 1977, A\&A, 54, 263

Ng, Y. K., Bertelli, G., Chiosi, C., \& Bressan, A. 1996, A\&A, 310,771

Orsatti, A. M., Vega, E., \& Marraco, H. G. 1998, AJ, 116, 266

Patat, F., \& Carraro, G. 1995, A\&AS, 114, 281

Piatti, A. E. 1997, PASP, 109, 343

Piatti, A. E., Clariá, J. J., \& Abadi, M. G. 1995, AJ, 110, 2813

Piatti, A. E., Clariá, J. J., \& Bica, E. 1998, ApJS, 116, 263

Piatti, A. E., Clariá, J. J., \& Bica, E. 1999, MNRAS, 303, 65

Ruprecht, J. 1966, Bull. Astron. Czechoslovakia, 17, 33

Santos, J. F. C. Jr., \& Bica, E. 1993, MNRAS, 260, 915

Schmidt-Kaler, Th. 1982, in Landolt-Börnstein. Numerical Data and Functional Relationships in Science and Technology, New Series, group VI, vol. 2b, ed. K. Schaifers, \& H. H. Voigt (Berlin, Springer Verlag)

Sher, D. 1965, MNRAS, 129, 237

Straiz̃ys, V. 1990, Multicolor Stellar Photometry, Pachart Publ. House (Tucson, Arizona)

Straiz̃ys, V., Clariá, J. J., Piatti, A. E., \& Kazlauskas, A. 1994, Baltic Astron., 3, 199

Twarog, B. A., Ashman, K. M., \& Anthony-Twarog, B. J. 1997, AJ, 114, 2556

Walker, A. R. 1985, MNRAS, 213, 889 